\title{
Pharmaceutical Product Information
}

National Cancer Institute

\section{Source}

National Cancer Institute. Pharmaceutical Product Information. NCI Thesaurus. Code C42667.

A document defining content and form of the information that has to be supplied with or about a pharmaceutical product by or on behalf of the marketing authorization holder. The minimum information in the product information is that defined by WHOs sample product information sheet. In international setting according to WHOs requirements, the content of the product information is agreed between the marketing authorization holder and the drug authorization authorities at the time the market authorization is issued. 